Psychiatry, which is both a health science and a social science, is centrally placed to richly benefit from qualitative and quantitative research methods.

Nnamdi Nkire,

Clinical Research Fellow/Registrar, St Davnet's Hospital, Monaghan, Ireland

Osamede Edokpolo,

Department of Psychiatry,

St Elizabeth's Hospital, Washington DC,

USA.

Reference

1. Fogel J. Research as part of the career of a psychiatrist entering clinical practice. Psychiatric Bulletin 2009; 33: 269-272.

\section{A model for primary mental healthcare in Ireland}

Dear Editor - We read with interest Kierans and Byrne's paper and presentation of a model for primary mental health care in Ireland. ${ }^{1}$ We welcome the advancement of the debate on primary care mental health. We agree there is a need for further developments within primary care, and the model proposed has many benefits. However we can also see some difficulties and advise a more collaborative approach between primary and secondary care.

The primary care practitioners the authors refer to are similar to the graduate mental health workers, who have been introduced as part of the Improving access to psychological treatments (IAPTS) in the UK. There is evidence that GPs do not trust the graduate workers. ${ }^{2}$ Fletcher et $\mathrm{al}^{3}$ described how a collaborative approach would ensure the role of the graduate mental health worker was embedded into the service, but they described many problems in setting up the posts, with graduates often unsupported and the process not having managerial support. Farrand et al ${ }^{4}$ conducted a qualitative evaluation of the role, and concluded that early difficulties were linked to inappropriate referrals and lack of clear role definition. They advised it was a valuable addition to a stepped care approach to mental healthcare in primary care. However, one of the key determinants of the impact of enhanced primary care mental health resources is the extent and quality of integration and communication at the interface between primary and secondary care. Lester et $\mathrm{al}^{5}$ found that patients assigned mental health workers in primary care were more satisfied with their care than controls but outcome was no different. Tylee and Walker ${ }^{6}$ use this finding to emphasise that 'bolting on' extra resources to existing care strategies does not improve outcome. Tey argues for systemic change, using collaborative care to introduce a chronic disease model for mental illnesses.

There is a risk the model proposed may result in a greater burden on specialist mental health teams. Consultation liaison models, where a consultant psychiatrist attends the general practice every six to eight weeks, has been shown to improve the appropriateness of referrals and improve the detection of those with mental health problems. ${ }^{7}$ We have found these meetings can be used to inform GPs on the availability of community resources, including self help groups, and recommend that any quasi specialists in mental health would be incorporated into these meetings.
We would be interested in knowing of other practices throughout the country, where there is greater liaison between primary care and secondary care, or where mental health professionals are working in primary care.

Vincent Russell, Martina Kelly, Joint Chair ICGP CPsychl Forum for Mental Health in Primary Care Dublin, Ireland

References

1. Kierans J, Byrne M. Ir J Psych Med 2010; $27(3):$ 152-156

2. Bower $P$, Rowland N. Effectiveness and cost effectiveness of counselling in Primary Care. Cochrane Database of Systematic Reviews, Issue 1, 2009

3. Fletcher J, Gavin M, Harkness E, Gask L. A collaborative approach to embedding graduate primary care mental health workers in the UK National Health Service. Health \& Social Care in the Community; 2006; 16(5): 451-459

4. Farrand P, Duncan F, Byng R. Impact of graduate mental health workers upon primary care mental health: a qualitative study. Health \& Social Care in the Community 2007; 15(5): 486-488.

5. Lester $\mathrm{H}$, Freemantle $\mathrm{N}$, Wilson $\mathrm{S}$ et al. Cluster randomised controlled trial of the effectiveness of primary care mental health workers. Br J Gen Pract 2007; 57(536):196203.

6. Tylee A, Walker P. We need a chronic disease model for depression in primary care. B $J$ Gen Pract 2007; 57(538): 348-350.

7. Russell V. Liaison psychiatry in rural general practice. Ir J Psych Med 2003; 20(2): 65-68.

\section{New long-stay psychiatric in-patients: a comparison of UK and Irish national audit}

Dear Editor - The above mentioned interesting study by Daly and Walsh, on new long-stay Irish patients in 2006; which was published in Ir J Psych Med 2009; 26(3): 134-139. The author thanks Daly and Walsh for their efforts to report on the first national level Irish study on NLS psychiatric in-patients.

This letter aims to compare the Irish study with much cited UK audit by Lelliott in $1992^{1,2}$ so as to stimulate further discussion and promote further research.

The Irish study covered all the NLS psychiatric in-patients coming from catchment population of $\sim 4.4$ million (Ireland population census, 2006); while the UK audit 1992, covered; estimated population: 26\% of England; $7 \%$ of Scottland, $41 \%$ of Wales; $82 \%$ of Northern Ireland; served by 59 mental health services of NHS (total catchment population 15.2 million).

The UK study ( $\mathrm{n}=905$ ) was cross-sectional, by census; while Irish study not only identified (by census on $31 / 03 / 2006)$, described NLS sample $(n=460)$, but also surveyed it after one year reporting that over two thirds of NLS patients $(n=315)$ were still residing at psychiatric units or hospitals, and $20 \%,(n=64)$, had become old long-stay patients (stay five years and over).

The UK study included patients aged 16-64 years, staying over six months up to three years while the Irish study included patients aged 16 years and over (and had 40\% patients ( $n=185$ ), aged 65 or over), with stay of one year to less than five years.

It is interesting that the UK study included a lower limit of long-stay as six months (rather than the traditional one year or over, as in the Irish study). This was because many participating units had a small number of acute beds and stay over six months was undesirable there; while the upper limit of three years was chosen, as the six-month lower limit for length of 
stay partly due to evidence that after this period the likelihood of subsequent discharge levels off, ${ }^{3}$ and partly to limit the number of assessments that participants, all of whom were busy clinical psychiatrists, would have to conduct.

In the UK study, 249 patients (28\%) were in acute wards, $49(5 \%)$ in intensive care wards, $26(3 \%)$ in psycho-geriatric wards and the remainder (64\%) in some form of rehabilitation or continuing care ward (one-third acute setting; while two-thirds medium/long-stay setting). In the Irish study, 384 patients $(84 \%)$ were in psychiatric hospitals; $26(6 \%)$ in general hospital psychiatric units, $50(11 \%)$ in other services. It would be interesting if Daly and Walsh could clarify further about what proportion of Irish NLS were in acute settings and what proportion were in medium/ long term care setting.

The UK study used a version of the FACE Profile ${ }^{5}$ modified specifically for this study. It allowed for the collection of data in nine domains. The Irish study did not use such an instrument.

In both studies, NLS patients were predominantly; 1. Single, $63 \%$ ( $n=570$, UK study), 69\% $(n=319), 2$. Men, $58 \%(n=532)$, UK study, 58\% ( $n=265$, Irish study); 3 . Diagnosis of schizophrenia, $57 \%$ ( $n=517$, UK study), $42 \%$ ( $n=192$, Irish study); 4. Admission legal status, in the UK study, 32\% ( $n=327$, admitted formally (involuntary), under the Mental Health Act 1983; $25 \%(n=114)$ admitted involuntarily in Irish study.

Interestingly in the UK study $72 \%(n=648)$ were unemployed, but this is not reported in Irish study. Another interesting observation was, in UK study 30\%, $(n=267)$ patients were admitted 5-10 times previously and 20\% ( $n=177$ ) had had more than 10 admissions, while in Irish study, $81 \%(n=371)$, were re-admissions, while $19 \%$ $(n=89)$, were first admissions. It would be useful if Daly and Walsh report on these factors.

The UK study reports on primary as well as subsidiary (secondary) diagnoses, while the Irish study reports only primary diagnoses. The term 'dual diagnosis' is well established, and comorbid substance misuse in patients with serious mental illness like schizophrenia is linked with increased aggression. ${ }^{4}$

In the UK study 163 patients (18\%) had committed a serious act of violence, $102(11 \%)$ had shown other dangerous or criminal behaviour, and $23(3 \%)$ had been admitted previously to a special hospital. Also in $42 \%(n=383)$ patients had moderate or severe risk of self-neglect or other non-deliberate self-harm; for $20 \%(n=181)$, severe risk of deliberate self-harm. Assessors felt that over half of the patients $(53 \%$, $n=476$ ), would pose a moderate or severe risk of violent or self-destructive behaviour, were they to be discharged.

The above risks are not reported in the Irish study, though it can be argued that 25\% patients in lthe rish study were admitted involuntarily (implying that they were posing a significant risk to self or others).

Based on the study of socio-demographic and clinical factors in NLS patients the UK study reported two particular sub-groups:

1. Younger NLS patients (aged 18-34, $\mathrm{n}=311$ ) were, predominantly single, men with schizophrenia; $43 \%$ of these had a history of serious violence, dangerous behaviour or admission to a special hospital and over one-third were formally detained.
2. Older NLS patients (aged 55-67, $n=212$ ) were predominantly married or previously married women, more often with a diagnosis of affective disorder or dementia and with poor personal and social functioning; over half were at moderate or severe risk of non-deliberate self-harm.

The Irish study does not identify such sub-groups. The author suggests that Daly and Walsh have the data on various patient factors, and by using statistical tests it is possible to examine the co-rrelation between these factors to identify such sub-groups, which may have a bearing in their management.

In the UK study assessors thought that $61 \%$ of patients would be better placed in a non-hospital setting; $47 \%$ were thought to require a community-based residential setting, and of these over one-half were still in hospital because no suitable community placement was available.

The UK study reports that NLS patients were occupying $9 \%$ of all beds available to those aged 18-64, with a wide variation (from 1-23\%) between services (occupancy over $10 \%$ in 25\% services), 31\% of English NLS patients were housed on acute wards, causing pressure on acute beds.

It would be useful if Daly and Walsh reported on bed occupancy by NLS patients and also get treating clinicians views on the appropriateness and reasons behind the long-stay.

The UK study reports an average point prevalence of NLS as 6.1 per 100,000 , population, per year; significantly lower in England and Wales (5.6, s.d. = 3.2) than in Scotland and Northern Ireland (10.7,s.d. $=6.4$, ANOVA F ratio $=10.9$, $P<0.01$ ). and estimated rate of accumulation was 1.3 per 100,000 population, per year.

The Irish study does not report on point prevalence and rate of accumulation. Corresponding author estimates point prevalence of NLS of $\sim 10.5$ per year per 100,000 population ( 460 NLS in 2006/Irish population in 2006 of 4.4 million). Daly and Walsh estimated that 110 NLS patients accumulate in Irish psychiatric units/hospitals. Based on this value the estimated rate of accumulation is 110/4.4 3 NLS per year per 100,000 population.

The UK study concludes that many NLS patients remain in hospital because their residential needs are not met by existing community provision. The Irish study concludes on similar lines that unless current services are improved and extended as advocated in A Vision for Change, ${ }^{6}$ it will be a number of years before this population (NLS) leaves psychiatric hospitals and such hospitals can close because of the lack of suitable alternatives. In accordance with policy recommendations, the continuing requirement for the development of specialised rehabilitation services to cater for the broad needs of those who remain in psychiatric hospitals is of utmost importance.

Ajay Dixit,

SHO Psychiatry, St Mary's Day Hospital, Parnell Street, Thurles, Co Tipperary

References

1. Lelliott P, Wing J, Clifford P. A National Audit of New Long-Stay Psychiatric Patients I: Method and Description of the Cohort. Brit Psychiatr 1994; 165; 160-169.

2. Lelliott P, Wing J. A National Audit of new long-stay psychiatric patients II: Impact on services. Brit J Psychiat 1994; 165: 170-178

3. Clifford P, Charman A, Webb Y et al. Planning for community care: long-stay populations scheduled for rundown or closure. Brit J Psychiatr 1991; 158: 190-196.

4. Scott H, Johnson S, Menezes $P$ et al. Substance misuse and risk of aggression and offending among the severely mentally ill. Br J Psychiatry 1998; 172: 345-350.

5. Clifford P. FACE Profile. London: Research Unit, Royal College of Psychiatrists, 1993. 6. A Vision for Change - recommendations of the expert group on mental health policy. 2006. Health Research Board, Dublin. 